\title{
Finite Element Analysis, Simplified by Conformal Mapping, of AN AXISYMMETRIC Dielectric CONSTANT PROBE
}

\author{
N. L. Buck，G. P. Groff，R. L. Raper
}

\begin{abstract}
A finite element analysis was conducted to obtain theoretical capacitance values for an axisymmetric dielectric constant sensor applied to layers of lean and fatty tissues. The sensor was under study as a noninvasive tool for determining the fat content of beef cattle and carcasses. A conformal mapping performed on a radial cross-section of the probe greatly simplified the analysis by eliminating field singularities at the edges of the conductors and correcting large disparities in the sizes of the elements. This coordinate transformation required a modification of the stiffness formula used in the finite element analysis. Capacitance values with estimated errors under $0.015 \%$ were obtained with a mesh of under 2,000 nodes. These values were used to identify deficiencies in an equation, developed from experimental data, relating the probe output to tissue thicknesses and dielectric constants. Theoretical capacitance values were needed because experimental error was hindering the development of this equation. The information in this article will be of use to other researchers who are performing axially symmetric finite element analyses or who are interested in dielectric constant measurements on layered materials.
\end{abstract}

Keywords. Complex numbers, Conformal mapping, Dielectric constant, Finite element analysis, Instrumentation, Meat, Non-destructive testing, Thickness.

$\mathrm{T}$ he fat content of a beef animal is an important factor in determining its value. Adequate intramuscular fat (marbling) is required for beef to receive a high quality grade. Excess fat, however, is a waste material which is expensive to produce. The USDA Agricultural Research Service, Instrumentation and Sensing Laboratory, Beltsville, Maryland, has been investigating non-invasive sensors to measure the fat content of animals. One of these sensors is a dielectric constant probe which operates at 50 to $150 \mathrm{MHz}$, shown in figure 1 . When the probe is applied to the exterior of an animal, which is composed of layers of hide, fat, and muscle, the capacitance is a function of the thicknesses and dielectric constants of the layers of tissue penetrated by the probe's electric field. This capacitance can be expressed as an apparent dielectric constant, i.e., the dielectric constant of a uniform, semi-infinite material to which the probe could be applied to yield the same capacitance. If several probes with different field penetration patterns are used on

\footnotetext{
Article was submitted for publication in February 1998; reviewed and approved for publication by the Information \& Electrical Technologies Division of ASAE in February 1999. Presented as ASAE Paper No. 94-308.

This research was supported by the U. S. Department of Agriculture, Agricultural Research Service, and performed at the Instrumentation and Sensing Laboratory, Beltsville Agricultural Research Center, Beltsville, Maryland. Commercial products are mentioned for information only; such mention does not constitute an endorsement by the U.S. Department of Agriculture nor a preference over other similar products not mentioned.

The authors are Nelson L. Buck, ASAE Member Engineer, Electronic Engineer, USDA Grain Inspection, Packers and Stockyards Administration, Washington, D.C.; Glenn P. Groff, Electrical Engineer, formerly with USDA, ARS Instrumentation and Sensing Laboratory; and Randy Raper, ASAE Member Engineer, Agricultural Engineer, USDA, ARS National Soil Dynamics Laboratory, Auburn, Ala. Corresponding author: Nelson L. Buck, USDA GIPSA FGIS FMD, Stop 3631, 1400 Independence Ave., Washington, DC 20250-3631; voice: (202) 720-0271; fax: (202) 720-1015; e-mail: nbuck@fgisdc.usda.gov.
}

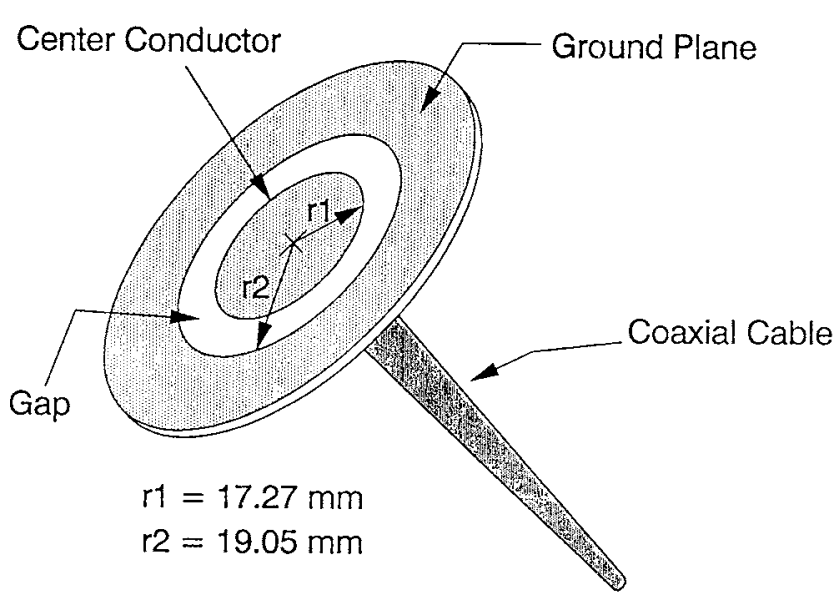

Figure 1-Coaxial dielectric constant probe for 50 to $150 \mathrm{MHz}$.

the same animal, it should be possible to determine the thickness and dielectric constant of each layer of tissue.

A formula was developed earlier for the apparent dielectric constant of a two-layer subject (Buck and Groff, 1995), which should be useful in determining the thickness of fatty tissue over a skinned carcass. However, the experiment from which this formula was derived contained too large an error for the accuracy of the formula to be determined. Such errors may make it impossible to develop formulas from experimental data for an instrument which must deal with the layers of hide, fat, and muscle on an intact animal. Theoretical values for the apparent dielectric constant seen by a sensor applied to a layered material were needed for further development of this technique. Finite element analysis is a practical method for obtaining such values. 
The probe has two features which make analysis difficult: (1) the sharp outer edge of the center conductor and the sharp inner edge of the ground plane create infinite voltage gradients; and (2) the electric field occupies an unbounded region of space. Such difficulties are sometimes eliminated by conformal mapping in two dimensions (Chang and Cheng, 1993; Kapoor and Schneider, 1995). Conformal mapping was also used to simplify this problem, although it was axisymmetric instead of twodimensional. The mathematics for doing so are developed in this article.

The dielectric constants of electrical conductors such as lean and fatty tissues are complex numbers. In this article, the term "dielectric constant" and the symbol $\varepsilon$ will refer to the relative complex dielectric constant, defined in the following equation (Institute of Electrical and Electronic Engineers, 1972; Hayt, 1989):

$$
\varepsilon=\varepsilon^{\prime}-\mathrm{j} \cdot \sigma /\left(2 \pi f \cdot \varepsilon_{0}\right)=\varepsilon^{\prime}-\mathrm{j} \cdot \varepsilon^{\prime \prime}
$$

where

$$
\begin{aligned}
& \varepsilon=\text { the complex dielectric constant } \\
& j=\sqrt{-1} \\
& \sigma=\text { the electrical conductivity of the material }(\mathrm{S} / \mathrm{m}) \\
& \mathrm{f}=\text { the frequency of the excitation signal }(\mathrm{Hz}) \\
& \varepsilon_{0}=\text { the permittivity of free space }(8.854187817 \mathrm{pF} / \mathrm{m}) \\
& \varepsilon^{\prime}=\text { the real part of the dielectric constant } \\
& \varepsilon^{\prime \prime}=\text { the loss index }=\sigma /\left(2 \pi f \cdot \varepsilon_{0}\right)
\end{aligned}
$$

\section{OBJECTIVES}

The objectives of this project were to:

1. Develop a finite element model to predict the capacitance of the dielectric constant probe.

2. Develop a procedure for simplifying an axisymmetric finite element problem by the use of conformal mapping.

3. Use the results of the finite element model to test the formula giving the capacitance of the probe applied to a sample consisting of two layers of different materials.

\section{PROBLEM FoRMULATION}

The capacitances of the front and rear surfaces of the probe appear in parallel. However, only the front surface electric field penetrates the sample being measured. The rear surface capacitance was considered an unknown constant.

The electric potential at any point in the vicinity of the probe was represented as $\mathrm{V} \cdot \mathrm{e}^{\mathrm{j} 2} \mathrm{ft}$, where $\mathrm{f}=$ frequency, $\mathrm{t}=$ time, and $\mathrm{V}$ is a complex voltage carrying both magnitude and phase information. Under the electrostatic approximation to Maxwell's equations, which is valid if the wavelength is long compared to the probe dimensions, the potential obeys Laplace's equation:

$$
\nabla \cdot\left[\varepsilon_{0} \cdot \varepsilon \cdot \nabla\left(\mathrm{V} \cdot \mathrm{e}^{\mathrm{j} 2 \pi \mathrm{ft}}\right)\right]=0
$$

Finite element analysis solves this equation by finding the unique voltage distribution which makes the partial derivative of the electric field energy with respect to the voltage at any point zero (Sylvester and Ferrari, 1990). The field energy $\mathrm{E}$ is:

$$
\mathrm{E}=0.5 \cdot \varepsilon_{0} \cdot \iiint \varepsilon \cdot \nabla \mathrm{V} \cdot \nabla \mathrm{V} \cdot\left(\mathrm{e}^{\mathrm{j} 2 \pi \mathrm{ft}}\right)^{2} \cdot \mathrm{dx} \cdot \mathrm{dy} \cdot \mathrm{dz}
$$

This field energy is proportional to the probe capacitance C:

$$
E=0.5 \cdot C \cdot\left(V_{1} \cdot e^{j 2 \pi f t}\right)^{2}
$$

where $\mathrm{V}_{1} \cdot \mathrm{e}^{\mathrm{j} 2 \pi \mathrm{ft}}$ is the voltage applied to the center conductor. Combining equations 3 and 4 gives $\mathrm{C}$ as a function of the voltage distribution in the space around the probe:

$$
\mathrm{C}=\left(\varepsilon_{0} / \mathrm{V}_{1}^{2}\right) \cdot \iiint \varepsilon \cdot \nabla \mathrm{V} \cdot \nabla \mathrm{V} \cdot \mathrm{dx} \cdot \mathrm{dy} \cdot \mathrm{dz}
$$

The factors that convert $\mathrm{C}$ to $\mathrm{E}$ are not functions of the spatial coordinates. Therefore the finite element analysis can work with $\mathrm{C}$ instead of $\mathrm{E}$ and determine the capacitance directly. The capacitance is independent of the magnitude of the applied voltage, so $V_{1}$ was set to 1 to simplify the equation.

Since the dielectric constant probe, shown in figure 1 , is symmetric about an axis through its center, the problem reduces to analyzing a radial cross-section, shown in figure 2. For this purpose, equation 5 was written in cylindrical coordinates $(\mathrm{r}, \theta, \mathrm{z})$ :

$$
\mathrm{C}=\varepsilon_{0} \cdot \iiint \varepsilon \cdot \nabla \mathrm{V} \cdot \nabla \mathrm{V} \cdot \mathrm{r} \cdot \mathrm{dr} \cdot \mathrm{d} \theta \cdot \mathrm{dz}
$$

where

$$
\nabla \mathrm{V} \cdot \nabla \mathrm{V}=(\partial \mathrm{V} / \partial \mathrm{r})^{2}+(1 / \mathrm{r})^{2} \cdot(\partial \mathrm{V} / \partial \theta)^{2}+(\partial \mathrm{V} / \partial \mathrm{z})^{2}
$$

Since $\partial \mathrm{V} / \partial \theta=0$, equation 6 was reduced to two dimensions by integrating with respect to $\theta$ from 0 to $2 \pi$ :

$$
\mathrm{C}=2 \pi \cdot \varepsilon_{0} \cdot \iint \varepsilon \cdot\left[(\partial \mathrm{V} / \partial \mathrm{r})^{2}+(\partial \mathrm{V} / \partial \mathrm{z})^{2}\right] \cdot \mathrm{r} \cdot \mathrm{dr} \cdot \mathrm{dz}
$$

The domain of integration of equation 7 is $0 \leq \mathrm{r}<\infty$ and $0 \leq \mathrm{z}<\infty$. Referring to figure 2 , the boundary along the $\mathrm{z}$-axis is a flux line. The boundary along the r-axis is an equipotential with an applied voltage from $r=0$ to $r=r 1$, corresponding to the center conductor of the probe; a flux line from $r=r 1$ to $r=r 2$, corresponding to the gap; and a grounded equipotential for $r>r 2$, corresponding to the ground plane of the probe. The capacitance obtained was that of an idealized probe with an infinite ground plane, which could be expected to be slightly smaller than that of the physical probe.

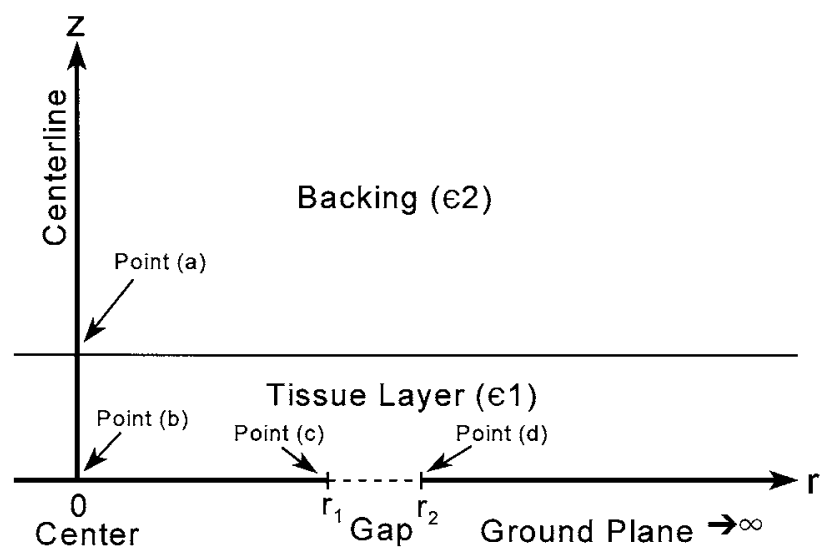

Figure 2-Radial cross-section of the probe. 


\section{SiMPLIFICATION OF PROBLEMS BY CONFORMAL MAPPING}

No finite element software available to the authors at the time of this study could analyze this probe because of the following complications: (1) the dielectric constants involved were complex numbers; and (2) an enormous number of elements of widely varying sizes was required because of the infinite voltage gradients at the sharp edges of the central disc and the ground plane, the small size of the gap and the first tissue layer, and the unlimited extent of the electric field in the surrounding space.

Chang and Cheng (1993) used conformal mapping to eliminate the last complication in their analysis of microstrip lines which, because they could be treated as infinitely long, obeyed Laplace's equation in two dimensions. Conformal mapping caused infinite voltage gradients located at critical points of the coordinate transformation to become finite in the new coordinate system and distances far from the field source to be compressed. No modifications to the finite element software were required, because a system that obeys Laplace's equation in two dimensions before transformation continues to do so afterward (Churchill, 1960).

While an axisymmetric problem can also be reduced to two dimensions (r, z), it does not obey Laplace's equation in two dimensions. However, as shown below, a conformal mapping can be applied to an axisymmetric problem and a finite element analysis performed successfully if we have the ability to change stiffness formulas in the finite element software.

Conformal mapping is applied in the following four steps (Churchill, 1960):

1. Combine the original coordinates $\mathrm{r}$ and $\mathrm{z}$ into the complex number $\mathrm{P}=\mathrm{r}+\mathrm{j} \cdot \mathrm{z}$

2. Combine the transform coordinates $x$ and $y$ into the complex number $\mathrm{W}=\mathrm{x}+\mathrm{j} \cdot \mathrm{y}$.

3. Relate $\mathrm{W}$ to $\mathrm{P}$ by an analytic function.

4. Re-write the capacitance in terms of the new coordinates $\mathrm{x}$ and $\mathrm{y}$.

Since the two sets of coordinates are related by an analytic function, the following relationships hold (Churchill, 1960):

$$
\begin{gathered}
\mathrm{dW} / \mathrm{dP}=\mathrm{Ro} \cdot \mathrm{ej} \varphi=\partial \mathrm{x} / \partial \mathrm{r}+\mathrm{j} \cdot \partial \mathrm{y} / \partial \mathrm{r}=\partial \mathrm{y} / \partial \mathrm{z}-\mathrm{j} \cdot \partial \mathrm{x} / \partial \mathrm{z}(8) \\
\mathrm{dP} / \mathrm{dW}=1 /(\mathrm{dW} / \mathrm{dP})=\mathrm{Ro}^{-1} \cdot \mathrm{e}^{-j \varphi}=\partial \mathrm{r} / \partial \mathrm{x}+\mathrm{j} \cdot \partial \mathrm{z} / \partial \mathrm{x} \\
=\partial \mathrm{z} / \partial \mathrm{y}-\mathrm{j} \cdot \partial \mathrm{r} / \partial \mathrm{y} \\
\partial \mathrm{x} / \partial \mathrm{r}=\partial \mathrm{y} / \partial \mathrm{z}=\operatorname{Ro} \cdot \cos (\varphi) \\
\partial \mathrm{y} / \partial \mathrm{r}=-\partial \mathrm{x} / \partial \mathrm{z}=\operatorname{Ro} \cdot \sin (\varphi) \\
\partial \mathrm{r} / \partial \mathrm{x}=\partial \mathrm{z} / \partial \mathrm{y}=\cos (\varphi) / \mathrm{Ro} \\
\partial \mathrm{r} / \partial \mathrm{y}=-\partial \mathrm{z} / \partial \mathrm{x}=\sin (\varphi) / \mathrm{Ro}
\end{gathered}
$$

In these equations, Ro is the magnification factor and $\varphi$ is the angle of rotation. The Jacobian of the transformation is:

$$
\partial(\mathrm{r}, \mathrm{z}) / \partial(\mathrm{x}, \mathrm{y})=\partial \mathrm{r} / \partial \mathrm{x} \cdot \partial \mathrm{z} / \partial \mathrm{y}-\partial \mathrm{r} / \partial \mathrm{y} \cdot \partial \mathrm{z} / \partial \mathrm{x}=1 / \mathrm{Ro}^{2}(14)
$$

So the capacitance integrated in the new coordinates is:

$$
\begin{gathered}
\mathrm{C}=\left(2 \pi \cdot \varepsilon_{0}\right) \cdot \iint \varepsilon \cdot\left[(\partial \mathrm{V} / \partial \mathrm{r})^{2}\right. \\
\left.+(\partial \mathrm{V} / \partial \mathrm{z})^{2}\right] \cdot \mathrm{r} \cdot\left(1 / \mathrm{Ro}^{2}\right) \cdot \mathrm{dx} \cdot \mathrm{dy}
\end{gathered}
$$

The partial derivatives of the voltage are still expressed in terms of $r$ and $z$. However, the chain rule for partial derivatives gives:

$$
\begin{aligned}
& \partial \mathrm{V} / \partial \mathrm{r}=\partial \mathrm{V} / \partial \mathrm{x} \cdot \partial \mathrm{x} / \partial \mathrm{r}+\partial \mathrm{V} / \partial \mathrm{y} \cdot \partial \mathrm{y} / \partial \mathrm{r} \\
& =[\partial \mathrm{V} / \partial \mathrm{x} \cdot \cos (\varphi)+\partial \mathrm{V} / \partial \mathrm{y} \cdot \sin (\varphi)] \cdot \mathrm{Ro} \\
& \partial \mathrm{V} / \partial \mathrm{z}=\partial \mathrm{V} / \partial \mathrm{y} \cdot \partial \mathrm{y} / \partial \mathrm{z}+\partial \mathrm{V} / \partial \mathrm{x} \cdot \partial \mathrm{x} / \partial \mathrm{z} \\
& =[\partial \mathrm{V} / \partial \mathrm{y} \cdot \cos (\varphi)-\partial \mathrm{V} / \partial \mathrm{x} \cdot \sin (\varphi)] \cdot \mathrm{Ro}
\end{aligned}
$$

Squaring equations 16 and 17 and adding them gives:

$$
(\partial \mathrm{V} / \partial \mathrm{r})^{2}+(\partial \mathrm{V} / \partial \mathrm{z})^{2}=\left[(\partial \mathrm{V} / \partial \mathrm{x})^{2}+(\partial \mathrm{V} / \partial \mathrm{y})^{2}\right] \cdot \mathrm{Ro}^{2}(18)
$$

Thus the capacitance can be expressed completely in the new coordinates as:

$$
\begin{aligned}
\mathrm{C}= & \left(2 \pi \cdot \varepsilon_{0}\right) \cdot \iint \varepsilon \cdot\left[(\partial \mathrm{V} / \partial \mathrm{x})^{2}+(\partial \mathrm{V} / \partial \mathrm{y})^{2}\right] \\
& \times \mathrm{Ro}^{2} \cdot \mathrm{r}(\mathrm{x}, \mathrm{y}) \cdot\left(1 / \mathrm{Ro}^{2}\right) \cdot \mathrm{dx} \cdot \mathrm{dy}
\end{aligned}
$$

The magnification factor Ro cancels out of the formula, leaving:

$$
\begin{aligned}
& \mathrm{C}=\left(2 \pi \cdot \varepsilon_{0}\right) \cdot \iint \varepsilon \cdot\left[(\partial \mathrm{V} / \partial \mathrm{x})^{2}\right. \\
& \left.+(\partial \mathrm{V} / \partial \mathrm{y})^{2}\right] \cdot \mathrm{r}(\mathrm{x}, \mathrm{y}) \cdot \mathrm{dx} \cdot \mathrm{dy}
\end{aligned}
$$

Thus the ONLY change required by the conformal mapping is to replace $\mathrm{r}$ by its expression in terms of $\mathrm{x}$ and $\mathrm{y}$.

\section{Conformal Mapping ApPlied to the Dielectric Constant Probe}

The analytic function which eliminates the infinite voltage gradients of the dielectric constant probe is (Churchill, 1960, Appendix 2 and pg 89):

$$
\mathrm{P}=0.5 \cdot(\mathrm{r} 2-\mathrm{r} 1) \cdot \sin (\mathrm{W})+0.5 \cdot(\mathrm{r} 2+\mathrm{r} 1)
$$

where $\mathrm{r} 1$ is the radius of the central disc, and $\mathrm{r} 2$ is the inner radius of the ground plane. Another way to write the same transformation is:

$$
\begin{gathered}
r=0.5(r 2-r 1) \cdot \sin (x) \cdot \cosh (y)+0.5(r 2+r 1) \\
z=0.5(r 2-r 1) \cdot \cos (x) \cdot \sinh (y)
\end{gathered}
$$

Inserting the above formula for $\mathrm{r}$ into equation 20 gives:

$$
\begin{aligned}
& \mathrm{C}=\pi \cdot \varepsilon_{0} \cdot \iint \varepsilon \cdot\left[(\partial \mathrm{V} / \partial \mathrm{x})^{2}+(\partial \mathrm{V} / \partial \mathrm{y})^{2}\right] \\
& \times[(\mathrm{r} 2-\mathrm{r} 1) \sin (\mathrm{x}) \cosh (\mathrm{y})+(\mathrm{r} 2+\mathrm{r} 1)] \cdot \mathrm{dx} \cdot \mathrm{dy}
\end{aligned}
$$


Factoring the constant $(\mathrm{r} 2+\mathrm{r} 1)$ out of the integral in equation 23 gives:

$$
\begin{aligned}
\mathrm{C}= & \pi \cdot(\mathrm{r} 2+\mathrm{r} 1) \cdot \varepsilon_{0} \cdot \iint \varepsilon \cdot\left[(\partial \mathrm{V} / \partial \mathrm{x})^{2}+(\partial \mathrm{V} / \partial \mathrm{y})^{2}\right] \\
& \times\left[\frac{\mathrm{r} 2-\mathrm{r} 1}{\mathrm{r} 2+\mathrm{r} 1} \sin (\mathrm{x}) \cosh (\mathrm{y})+1\right] \cdot \mathrm{dx} \cdot \mathrm{dy}
\end{aligned}
$$

This formula can be simplified by defining two constants $\mathrm{q} 1=\pi \cdot(\mathrm{r} 2+\mathrm{r} 1) \cdot \varepsilon_{0}$ and $\mathrm{q} 2=(\mathrm{r} 2-\mathrm{r} 1) /(\mathrm{r} 2+\mathrm{r} 1)$. Then equation 24 becomes:

$$
\begin{aligned}
\mathrm{C} & =\mathrm{q} 1 \cdot \iint \varepsilon \cdot\left[(\partial \mathrm{V} / \partial \mathrm{x})^{2}+(\partial \mathrm{V} / \partial \mathrm{y})^{2}\right] \\
& \times[1+\mathrm{q} 2 \cdot \sin (\mathrm{x}) \cosh (\mathrm{y})] \cdot \mathrm{dx} \cdot \mathrm{dy}
\end{aligned}
$$

Figure 2 shows the dielectric constant probe in the $(\mathrm{r}, \mathrm{z})$ system, applied to a two-layer sample. Figure 3 shows the probe in the $(x, y)$ system. The field singularities in the original problem are finite in the transformed problem because they coincide with the critical points of the transformation at $(x, y)=( \pm \pi / 2,0)$. The point $(-\pi / 2,0)$ corresponds to the edge of the center conductor at $(\mathrm{r}, \mathrm{z})=$ $(\mathrm{r} 1,0)$. The point $(\pi / 2,0)$ corresponds to the inner edge of the ground plane at $(r, z)=(r 2,0)$. The $\mathrm{X}$-axis between $-\pi / 2$ and $\pi / 2$ corresponds to the gap between the conductors. The transformation folds the center conductor and the ground plane parallel to the y-axis. The centerline of the probe and the boundary between the dielectrics become curved.

In addition to eliminating the singularities in the original problem, the coordinate transformation enlarges the region near the gap between the conductors and compresses regions far from the gap logarithmically. This space

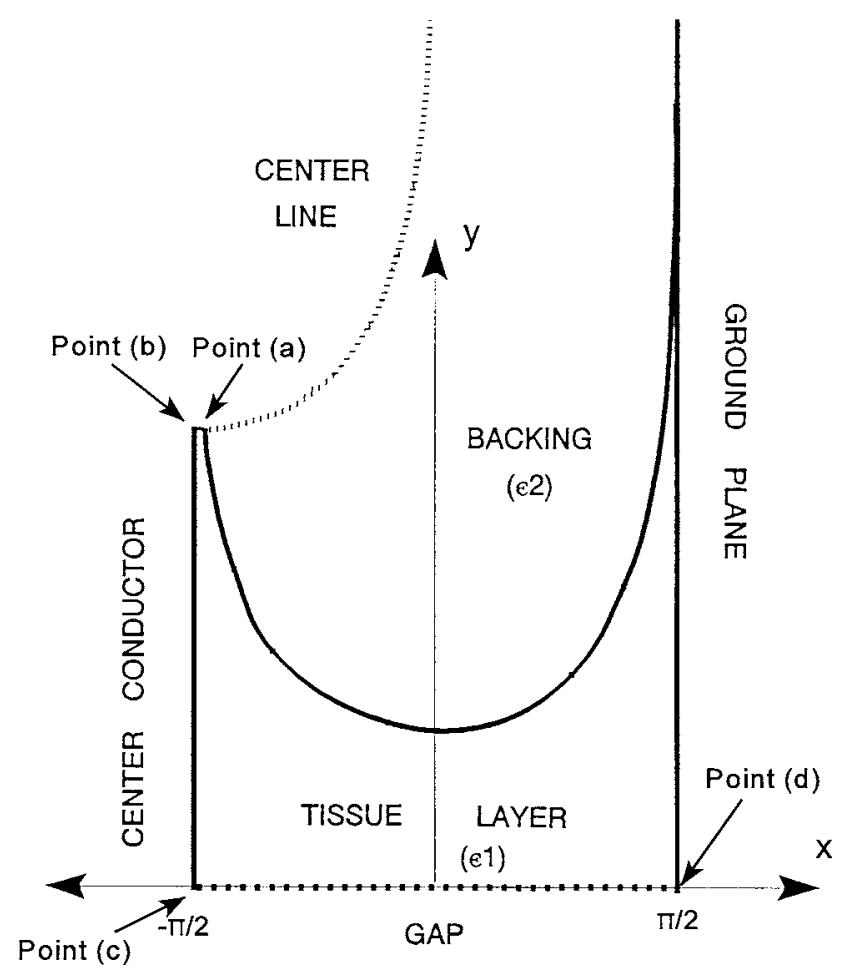

Figure 3-Probe transformed by conformal mapping. distortion makes the energy density more uniform in the transformed coordinates and greatly facilitates mesh generation. It also allows fairly small rectangular elements at large y values to represent enormous regions of space far from the probe.

\section{PROGRAM}

Although conformal mapping simplified the mesh and eliminated the infinite voltage gradients, it still did not enable the software available to the researchers to analyze the probe, so a new finite element program was written in Microsoft Fortran. This program allowed complex dielectric constants and, to allow the use of transformed coordinates, allowed the energy function to be modified. The program allowed the problem domain to be divided into quadratic elements with one node at each corner and one in the middle of each side. The curved sides possible with such elements provided a good fit to the curved boundaries in the transformed problem. Quadratic elements also converge to the correct answer more quickly than linear elements as the mesh is refined (Sylvester and Ferrari, 1990). Both triangular and parallelogram elements were used. A parallelogram was required to have straight sides, while a triangle was allowed one curved side. Parallelograms were used wherever possible, in order to reduce the total number of elements and allow faster program execution.

When the space around the probe is divided into elements, the integral giving the capacitance $C$ (eq. 25) can be expressed as a sum of integrals performed on the individual elements:

$$
\mathrm{C}=\sum_{\mathrm{e}=1}^{\mathrm{ne}} \mathrm{C}_{\mathrm{e}}
$$

where

$$
\begin{gathered}
\mathrm{C}_{\mathrm{e}}=\mathrm{q} 1 \cdot \varepsilon_{\mathrm{e}} \cdot \iint\left[(\partial \mathrm{V} / \partial \mathrm{x})^{2}+(\partial \mathrm{V} / \partial \mathrm{y})^{2}\right] \\
\quad \times[1+\mathrm{q} 2 \cdot \sin (\mathrm{x}) \cosh (\mathrm{y})] \cdot \mathrm{dx} \cdot \mathrm{dy}
\end{gathered}
$$

Here ne $=$ the total number of elements, and the integral $\mathrm{C}_{\mathrm{e}}$ in equation 14 is performed only over element e. The dielectric constant $\varepsilon_{\mathrm{e}}$ is taken out of the integral because it is constant over element e. To assure that $\varepsilon_{\mathrm{e}}$ was indeed a constant for all elements, the boundary lines between materials with different dielectric constants were made to coincide with element boundaries.

The voltage distribution within element $e$ is approximated by:

$$
\mathrm{V}(\mathrm{x}, \mathrm{y})=\Sigma_{\mathrm{n}} \mathrm{V}_{\mathrm{n}} \cdot \mathrm{L}_{\mathrm{n}, \mathrm{e}}(\mathrm{x}, \mathrm{y})
$$

where

$$
\begin{aligned}
\mathrm{V}_{\mathrm{n}} & =\text { the voltage at node } \mathrm{n} \\
\mathrm{L}_{\mathrm{n}, \mathrm{e}}(\mathrm{x}, \mathrm{y})= & \text { the shape function for node } \mathrm{n} \text { within } \\
& \text { element } \mathrm{e}
\end{aligned}
$$

Shape functions are described by Sylvester and Ferrari (1990). They are defined so $\mathrm{L}_{\mathrm{n}, \mathrm{e}}(\mathrm{x}, \mathrm{y})=1$ at node $\mathrm{n}$, $\mathrm{L}_{\mathrm{n}, \mathrm{e}}(\mathrm{x}, \mathrm{y})=0$ at all other nodes and everywhere outside of element e, and $\mathrm{V}(\mathrm{x}, \mathrm{y})$ is continuous across the boundaries between elements. Performing the operations in 
equation 27 using the approximate voltage distribution (eq. 28) converts equation 27 to:

$$
\mathrm{C}_{\mathrm{e}}=\Sigma_{\mathrm{n}} \Sigma_{\mathrm{m}} \mathrm{V}_{\mathrm{n}} \cdot \mathrm{V}_{\mathrm{m}} \cdot \mathrm{S}_{\mathrm{n}, \mathrm{m}, \mathrm{e}}
$$

where $S_{n, m, e}$ is a constant called the "local stiffness" between nodes $\mathrm{n}$ and $\mathrm{m}$ in element e. When the $\mathrm{C}_{\mathrm{e}} \mathrm{s}$ are summed to obtain $\mathrm{C}$, the formula becomes:

$$
\mathrm{C}=\Sigma_{\mathrm{n}} \Sigma_{\mathrm{m}} \mathrm{V}_{\mathrm{n}} \cdot \mathrm{V}_{\mathrm{m}} \cdot \mathrm{S}_{\mathrm{n}, \mathrm{m}}
$$

where $S_{n, m}$ is the stiffness between nodes $n$ and $m$. If nodes $\mathrm{n}$ and $\mathrm{m}$ both appear on two elements e1 and e2, then $\mathrm{S}_{\mathrm{n}, \mathrm{m}}=\mathrm{S}_{\mathrm{n}, \mathrm{m}, \mathrm{e} 1}+\mathrm{S}_{\mathrm{n}, \mathrm{m}, \mathrm{e} 2}$. In matrix form, equation 30 is:

$$
\mathrm{C}=[\mathrm{V}]^{\prime} \cdot[\mathrm{S}] \cdot[\mathrm{V}]
$$

where [V] is the vector of node voltages, $[\mathrm{S}]$ is the stiffness matrix, and [V]' is the transpose of [V]. $\mathrm{S}_{\mathrm{n}, \mathrm{m}}=\mathrm{S}_{\mathrm{m}, \mathrm{n}}$, so [S] is symmetric.

If the known node voltages on the ground plane and center conductor are separated from the unknown voltages elsewhere, equation 31 becomes:

$$
\begin{gathered}
\mathrm{C}=[\mathrm{Vu}]^{\prime} \cdot[\mathrm{Suu}] \cdot[\mathrm{Vu}]+2 \cdot[\mathrm{Vu}]^{\prime} \cdot[\mathrm{Suk}] \cdot[\mathrm{Vk}] \\
+[\mathrm{Vk}]^{\prime} \cdot[\mathrm{Skk}] \cdot[\mathrm{Vk}] \text { or } \\
\mathrm{C}=[\mathrm{Vu}]^{\prime} \cdot[\mathrm{Suu}] \cdot[\mathrm{Vu}]+2 \cdot[\mathrm{Vu}]^{\prime} \cdot[\mathrm{B}]+\mathrm{C} 1
\end{gathered}
$$

where

$$
\begin{aligned}
{[\mathrm{Vu}]=} & \text { the unknown node voltages } \\
{[\mathrm{Vk}]=} & \text { the known node voltages } \\
{[\mathrm{Suu}]=} & \text { the matrix of stiffnesses between pairs of } \\
& \text { unknown node voltages } \\
{[\mathrm{Skk}]=} & \text { the matrix of stiffnesses between pairs of known } \\
& \text { node voltages } \\
{[\mathrm{Suk}]=} & \text { the matrix of stiffnesses between known and } \\
& \text { unknown voltages } \\
{[\mathrm{B}]=} & {[\mathrm{Suk}] \cdot[\mathrm{Vk}]=\text { a vector of known constants } } \\
\mathrm{C} 1= & {[\mathrm{Vk}]^{\prime} \cdot[\mathrm{Skk}] \cdot[\mathrm{Vk}]=\text { a known constant } }
\end{aligned}
$$

The problem was solved by setting the partial derivatives of $\mathrm{C}$ with respect to all the unknown node voltages equal to zero and solving the resulting system of linear equations for the voltages:

$$
\partial \mathrm{C} / \partial[\mathrm{Vu}]=[\mathrm{Vu}]^{\prime} \cdot[\mathrm{Suu}]+[\mathrm{Suu}] \cdot[\mathrm{Vu}]+2 \cdot[\mathrm{B}]=0
$$

The matrix [Suu] is symmetric, so equation 20 reduces to:

$$
[\mathrm{Suu}] \cdot[\mathrm{Vu}]=-[\mathrm{B}]
$$

Both $\mathrm{C} 1$ and [B] were built up while the stiffnesses were being calculated. The stiffness matrix [Suu] is a symmetric, banded matrix. Modifications of the LINPACK subroutines ZGBFA and ZGBSL (Dongarra et. al., 1979) were used to solve the equations to obtain [Vu]. Substituting $[\mathrm{Suu}] \cdot[\mathrm{Vu}]=-[\mathrm{B}]$ into equation 32 gives:

$$
\mathrm{C}=[\mathrm{Vu}]^{\prime} \cdot[\mathrm{B}]+\mathrm{C} 1
$$

or

$$
\mathrm{C}=\mathrm{C} 1-[\mathrm{B}]^{\prime} \cdot[\mathrm{Suu}]^{-1} \cdot[\mathrm{B}]
$$

\section{ERROR ESTIMATION}

The calculated capacitance has four error components:

1. Roundoff error is caused by the limited precision of the computer. This error was minimized by doing all calculations in double precision.

2. Stiffness approximation error is caused by the use of numerical integration to calculate stiffnesses. Within a straight-sided parallelogram element, stiffnesses were calculated by integrating the product of a polynomial with $\sin (x) \cdot \cosh (y)$. This was done by a power series approximation to an accuracy of about one part in $10^{15}$. For a triangular element with one curved side, $\sin (\mathrm{x}) \cdot \cosh (\mathrm{y})$ was multiplied by a rational function with a linear denominator and a 36-point Gauss-Legendre integration was performed.

3. Discretization error is caused by the true voltage distribution not being a quadratic polynomial. This error is theoretically proportional to the fourth power of the linear dimensions of the elements (Sylvester and Ferrari, 1990).

4. Field truncation error is caused by the inability of the program to model fields extending to infinity. This error was minimized by extending the model to $\mathrm{y}=7$, which corresponds to a shell around the probe at a distance of $0.487 \mathrm{~m}$. The size of the error was estimated by comparing the capacitance calculated with the line at $y=7$ grounded to the capacitance calculated with the same line ungrounded. The difference was approximately $0.01 \%$.

The approximation and discretization errors both decrease as the mesh is refined. However, solving the simultaneous equations magnifies the effects of the roundoff and stiffness approximation errors. As the mesh is refined, the equation set increases in size and becomes more ill-conditioned, increasing the error magnification. If the mesh is made too fine, the result may no longer converge to the correct answer.

\section{Mesh Generation}

A semi-automatic mesh generator was written for this program. Nodes were considered to be arranged in rows and columns. The operator specified the starting $y$-value of each row and the starting $\mathrm{x}$-value of each column. Where the rows and columns were curved, the operator specified an equation for the curve. The mesh generator created elements in the spaces between the intersecting rows and columns. Where the sides of the elements were straight and parallel, the elements were parallelograms. Otherwise, the elements were triangular. As much as possible, the elements were made uniform in size and square in shape. The mesh was refined manually by increasing the number of rows and columns, keeping the elements of the finer mesh similar in shape to those of the coarser mesh. Figures 4 and 5 show two meshes generated by this procedure.

An equipotential boundary condition was created by assigning a known voltage to the nodes on that boundaries. Any boundary of the finite element model which was not 
assigned a voltage automatically became a flux line. This is known as a natural boundary condition (Sylvester and Ferrari, 1990).

\section{ANALYSIS OF A SYSTEM WITH KNOWN CAPACITANCE}

Finite element analysis is a well-established procedure, and equations 8-25 demonstrate that the analysis of a problem after conformal mapping is mathematically identical to the analysis of the original problem. This technique therefore does not require an extensive validation. However, in order to rule out errors in the mathematical analysis and programming mistakes that might produce incorrect answers, the finite element

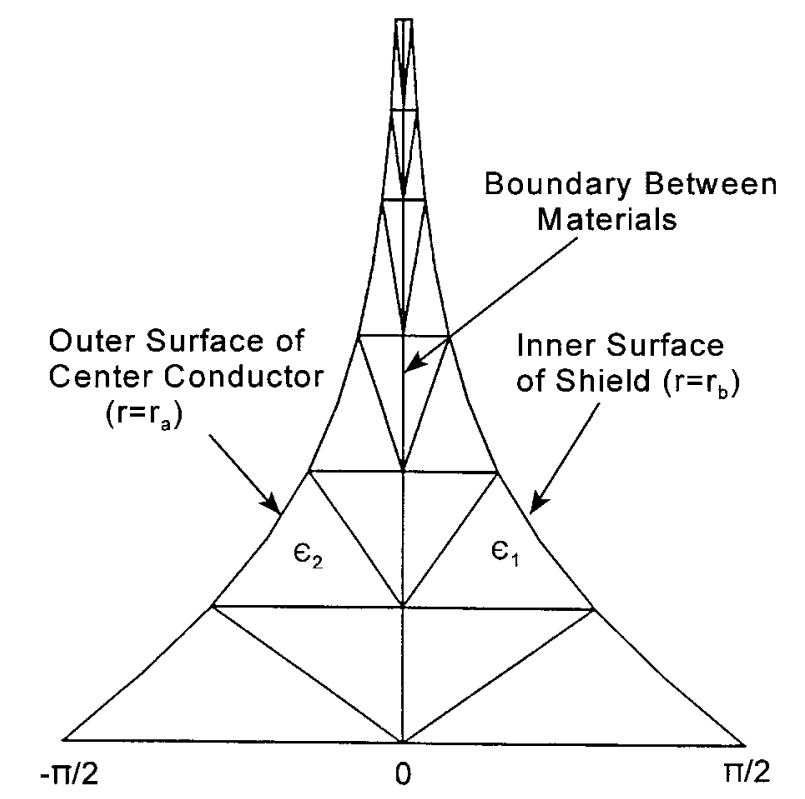

Figure 4-Coaxial cable transformed by conformal mapping, with mesh.

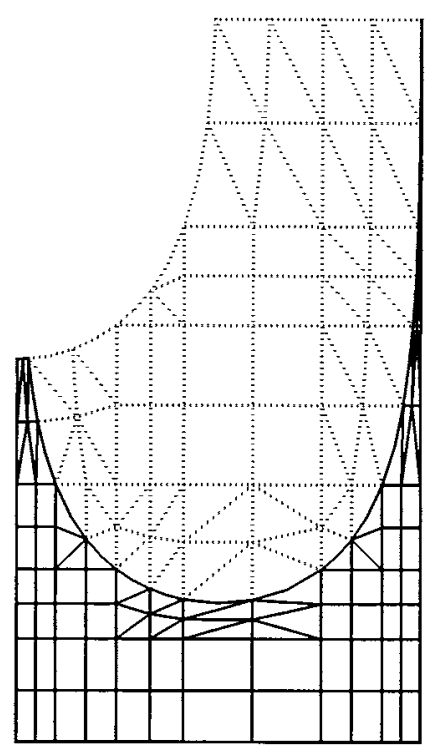

Figure 5-Transformed probe with finite element mesh. program and the conformal mapping were used to determine the known capacitance of a hypothetical length of coaxial cable before being used for the dielectric constant probe. This transformation complicated the field geometry of the cable rather than simplifying it. It would not have been used if the object had actually been to analyze the cable. To avoid end effects, the length of cable analyzed was considered to be part of an infinitely long cable. The capacitance of such a length of cable with air as the dielectric is (Ramo et al., 1967):

$$
\mathrm{C}=2 \pi \cdot \mathrm{L} \cdot \varepsilon_{0} \cdot \varepsilon / \ln \left(\mathrm{r}_{\mathrm{b}} / \mathrm{r}_{\mathrm{a}}\right)
$$

where

$$
\begin{aligned}
& \mathrm{C}=\text { the capacitance }=30.09094 \mathrm{pF} \\
& \mathrm{r}_{\mathrm{a}}=\text { radius of the center conductor }=25.4 \mathrm{~mm}(1 \mathrm{in} .) \\
& \mathrm{r}_{\mathrm{b}}=\text { the inner radius of the shield }, \mathrm{r}_{\mathrm{b}} / \mathrm{r}_{\mathrm{a}}=2.30230 \\
& \mathrm{~L}=\text { the length of the cable }=0.4510523 \mathrm{~m}(17.758 \mathrm{in} .) \\
& \varepsilon=\text { the dielectric constant of the insulation }=1.0
\end{aligned}
$$

The same conformal mapping used for the probe was applied to the cable, with $r_{1}=r_{a}$ and $r_{2}=r_{b}$. Thus at $y=0$, the outer surface of the center conductor and the inner surface of the shield passed through the critical points at $x= \pm \pi / 2$. The cable length $L$ was selected by letting $y$ vary from 0 to 4 along the two conductors, producing $\mathrm{z}$ values of 0 to $0.4510523 \mathrm{~m}$. Figure 4 shows the transformed cable with a mesh superimposed. Solutions were obtained for progressively finer meshes, giving the capacitance values shown in table 1 . The values converged to the theoretical value, with an error approximately proportional to the fourth power of the element size. This is the rate of convergence predicted by theory if discretization error dominates (Sylvester and Ferrari, 1990).

Table 1. Errors in the capacitance of a coaxial cable with an air dielectric calculated by finite element analysis

\begin{tabular}{lcc}
\hline Relative Element Size & Capacitance $(\mathrm{pF})$ & Error $(\mathrm{pF})$ \\
\hline 1 & 30.44243 & 0.35146 \\
$1 / 2$ & 30.11791 & 0.02694 \\
$1 / 4$ & 30.09286 & 0.00189 \\
$1 / 8$ & 30.09100 & 0.00006 \\
Theoretical value & 30.09094 & \\
\hline
\end{tabular}

As a further check, the problem was complicated by considering the cable to be filled with a two-layer dielectric consisting of fatty tissue with a dielectric constant $\varepsilon_{1}=8-$ 9.1.j for $\mathrm{r}>\mathrm{r}_{\mathrm{c}}$ and lean tissue with a dielectric constant $\varepsilon_{2}=$ $66-120 \cdot \mathrm{j}$ for $\mathrm{r}<\mathrm{r}_{\mathrm{c}}$. This case is equivalent to two coaxial capacitors in series:

$$
\mathrm{C}=2 \pi \cdot \mathrm{L} \cdot \varepsilon_{0} /\left[\left(1 / \varepsilon_{1}\right) \cdot \ln \left(\mathrm{r}_{\mathrm{b}} / \mathrm{r}_{\mathrm{c}}\right)+\left(1 / \varepsilon_{2}\right) \cdot \ln \left(\mathrm{r}_{\mathrm{c}} / \mathrm{r}_{\mathrm{a}}\right)\right]
$$

Substituting the values of the variables gives $\mathrm{C}=$ 518.37079 - j.620.96684. Table 2 shows the results of the finite element analysis with progressively finer meshes. Again, the capacitance from the finite element analysis approached the theoretical value with an error proportional to the fourth power of element size. 
Table 2. Errors in the calculated capacitance of a hypothetical coaxial cable filled with layers of conductive dielectric representing lean and fat tissue

\begin{tabular}{lll}
\hline Relative Element Size & Capacitance $(\mathrm{pF})$ & Error $(\mathrm{pF})$ \\
\hline 1 & $521.86654-624.88680 \mathrm{j}$ & $3.49575-3.91996 \mathrm{j}$ \\
$1 / 2$ & $518.64852-621.27890 \mathrm{j}$ & $0.27774-0.31207 \mathrm{j}$ \\
$1 / 4$ & $518.39034-620.98871 \mathrm{j}$ & $0.01955-0.02188 \mathrm{j}$ \\
$1 / 8$ & $518.37209-620.96829 \mathrm{j}$ & $0.00130-0.00145 \mathrm{j}$ \\
Theoretical value & $518.37079-620.96684 \mathrm{j}$ & \\
\hline
\end{tabular}

\section{RESULTS}

After the model was checked against a length of coaxial cable, the dielectric constant probe of figure 1 was analyzed. The probe was assumed to be covered by a layer of material $1.587 \mathrm{~mm}$ (1/16 in.) thick with a dielectric constant $\varepsilon_{1}$, backed by a semi-infinite thickness of a second material with dielectric constant $\varepsilon_{2}$. The center conductor radius $\left(\mathrm{r}_{1}\right)$ was $17.272 \mathrm{~mm}$ (0.68 in.), and the inner radius of the ground plane $\left(r_{2}\right)$ was $19.05 \mathrm{~mm}(0.75 \mathrm{in}$.). Figure 5 shows the transformed probe with a mesh superimposed. The elements in the second material are drawn with dotted lines.

Finite element analyses were performed repeatedly with different values of $\varepsilon_{1}$ and $\varepsilon_{2}$. For each combination, solutions were obtained with progressively finer meshes to obtain an error estimate. Table 3 gives the statistics for the meshes used. The fourth mesh in the series (1723 unknown node voltages) was the finest mesh used on all but one analysis. In the case where plastic was backed by lean, the result obtained with the coarsest mesh did not fall on the trend of the results obtained with the finer meshes, so a fifth mesh was included to verify that the results were indeed converging. The capacitance obtained with each mesh was plotted against the relative size of the elements raised to the power $(2,3$, or 4$)$ that made the plot linear. Then the difference between the capacitance obtained from the finest mesh and the capacitance obtained by extrapolating the plot to zero element size was added to the estimated field truncation error to yield the error estimate. Table 4 lists the probe capacitances obtained with the finest mesh. Figure 6 shows the variation in calculated capacitance with element size for a probe covered by a layer of fatty tissue $\left(\varepsilon_{1}=8-9.1 \mathrm{j}\right)$ backed by lean $\left(\varepsilon_{2}=\right.$ $66-120 \mathrm{j})$. The values converge with an error proportional to the fourth power of element size, indicating that discretization error dominates. Figure 7 shows the variation in calculated capacitance with element size for a probe covered with a sheet of plastic $\left(\varepsilon_{1}=2.5\right)$ backed by lean. Except for the coarsest mesh, the error in the imaginary part of $\mathrm{C}$ is approximately proportional to the fourth power of the element size, but the error in the real part is proportional to the square.

Table 3. Details of meshes used in the finite element analyses of the dielectric constant probe

\begin{tabular}{cccc}
\hline $\begin{array}{c}\text { Relative } \\
\text { Element } \\
\text { Size }\end{array}$ & $\begin{array}{c}\text { Number of } \\
\text { Unknown } \\
\text { Voltages }\end{array}$ & $\begin{array}{c}\text { Number of } \\
\text { Node } \\
\text { Voltages }\end{array}$ & $\begin{array}{c}\text { Number of } \\
\text { Known Node } \\
\text { Elements }\end{array}$ \\
\hline 1 & 109 & 22 & 46 \\
$1 / 2$ & 433 & 42 & 184 \\
$1 / 3$ & 971 & 62 & 414 \\
$1 / 4$ & 1723 & 82 & 736 \\
$1 / 5$ & & & \\
(one analysis only) & 2689 & 102 & 1150 \\
\hline
\end{tabular}

Table 4. Results of finite element analysis of the dielectric constant probe applied to different materials

\begin{tabular}{|c|c|c|c|c|}
\hline$\varepsilon_{1}$ & $\varepsilon_{2}$ & Capacitance $(\mathrm{pF})$ & $\begin{array}{l}\text { Estimated } \\
\text { Relative } \\
\text { Error }(\%)\end{array}$ & $\begin{array}{l}\text { Thickness } \\
\text { Function }[\mathrm{F}(\mathrm{t})]\end{array}$ \\
\hline 1 & 1 & 1.2447 & 0.015 & \\
\hline $66-120 j$ & 1 & $34.2524-60.5995 \mathrm{j}$ & 0.011 & $0.4231+0.0006 j$ \\
\hline $8-9.1 \mathrm{j}$ & 1 & $4.9503-4.6105 \mathrm{j}$ & 0.011 & $0.4279+0.0055 \mathrm{j}$ \\
\hline 2.6 & 1 & 2.0950 & 0.013 & 0.4816 \\
\hline $66-120 j$ & $8-9.1 \mathrm{j}$ & $40.5115-68.8522 j$ & 0.011 & $0.4305+0.0016 j$ \\
\hline $8-9.1 \mathrm{j}$ & $66-120 j$ & $27.4491-35.9672 \mathrm{j}$ & 0.012 & $0.5958-0.0079 \mathrm{j}$ \\
\hline 2.5 & $66-120 \mathrm{j}$ & $12.9291-1.4541 \mathrm{j}$ & 0.011 & $0.6269-0.0155 \mathrm{j}$ \\
\hline
\end{tabular}

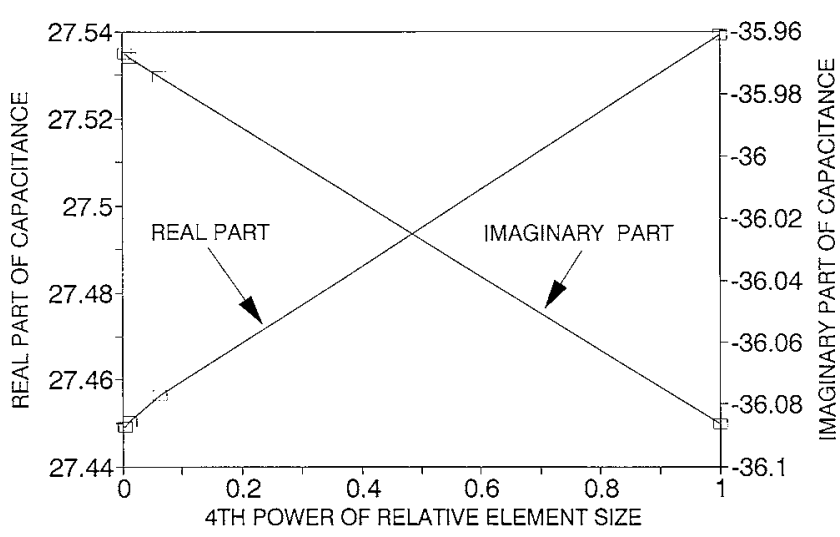

Figure 6-Variation of predicted capacitance with element size, fat backed by lean.

These results were used to evaluate a formula developed previously for the capacitance of a probe applied to a twolayer sample (Buck and Groff, 1995):

$$
C=C_{a} \cdot \varepsilon_{1} \cdot \frac{1-F(t) \cdot R_{12}}{1+F(t) \cdot R_{12}}
$$

where

$\mathrm{t}=$ the thickness of the top dielectric

$\mathrm{C}_{\mathrm{a}}=$ the capacitance of the probe in air

$\mathrm{R}_{12}=$ the electrostatic reflection coefficient $=\left(\varepsilon_{1}-\varepsilon_{2}\right) /$ $\left(\varepsilon_{1}+\varepsilon_{2}\right)$

$\mathrm{F}(\mathrm{t})=\mathrm{a}$ function of thickness which is 1 at $\mathrm{t}=0$ and 0 for $\mathrm{t}=\infty$

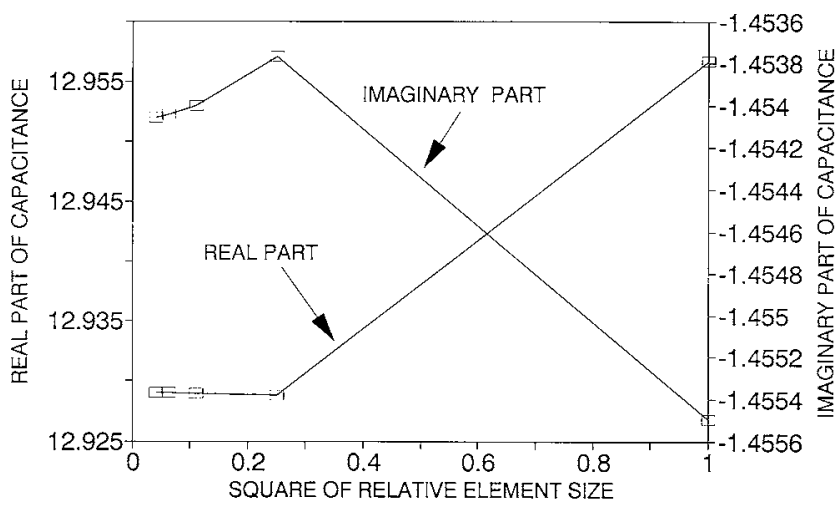

Figure 7-Variation of predicted capacitance with element size, plastic backed by lean. 
The thickness function $\mathrm{F}(\mathrm{t})$ is ideally real and independent of $\varepsilon_{1}$ and $\varepsilon_{2}$. Solving equation 39 for $F(t)$ gives:

$$
F(t)=\frac{C_{a} \cdot \varepsilon_{1}-C}{C_{a} \cdot \varepsilon_{1}+C} \frac{1}{R_{12}}
$$

The values obtained for $\mathrm{F}(\mathrm{t})$ using various values of $\varepsilon_{1}$ and $\varepsilon_{2}$ and their corresponding capacitances are shown in table 4 . The $\mathrm{F}(\mathrm{t})$ values vary about the mean by about $\pm 10 \%$ and have small imaginary parts which are within $1.3 \%$ of the real parts. $\mathrm{F}(\mathrm{t})$ is approximately a linear function of the electrostatic reflection coefficient $R_{12}$. The regression equation:

$$
F(t)=(0.5205-0.0050 j)-(0.1012-0.0036 j) \cdot R_{12}(41)
$$

fits the data in table 4 to within $1.7 \%$.

\section{DiSCUSSION AND CONCLUSIONS}

An axisymmetric finite element problem can be modified by conformal mapping, and in some cases the mapping may greatly simplify the problem. If such a transformation is performed, the radius coordinate $r$ in the integral which yields stiffness must be replaced by its expression in terms of the new coordinates. The particular mapping studied was integrable by a power series when the elements had straight sides. Curved sides required a numerical integration procedure which was more difficult, but not excessively so.

The capacitance of the front surface of an axisymmetric dielectric constant probe was determined with an estimated error of $0.015 \%$ or less by a combination of finite element analysis and a conformal mapping which eliminated the field singularities at the edges of the conducting plates. The conformal mapping also scaled the space around the probe logarithmically, expanding the region with the greatest field strength and allowing a small number of elements to model the space far from the probe. With the problem thus simplified, it was successfully analyzed by a finite element program and a simple semi-automatic mesh generator running on a desktop computer. In order to accommodate conformal mapping, the finite element program called userwritten subroutines to evaluate the stiffness integrals for the two types of elements: parallelogram elements with straight sides and triangular elements with one curved side. If a different conformal mapping is used, these subroutines must be re-written.

An empirical formula (eq. 39) for the capacitance of a probe applied to a two-layer sample (Buck and Groff, 1995) includes a function of the thickness of the top layer which had been assumed to be real and independent of the dielectric constants of the two materials. The finite element analyses showed that this thickness function does have an imaginary part, but that it is small enough $(1.3 \%)$ to be neglected in most measurements. The thickness function also showed a dependence on the dielectric constants of the materials involved which was too large to ignore $( \pm 10 \%)$. However, for the single thickness studied, the thickness function was well approximated by a linear function of the electrostatic reflection coefficient $\left(\varepsilon_{1}-\varepsilon_{2}\right) /\left(\varepsilon_{1}+\varepsilon_{2}\right)$.

At the time this analysis was performed, the simplifications achieved with conformal mapping made the analysis possible with the tools that were available. It may also be advantageous to simplify an axisymmetric problem with conformal mapping even when the tools are available to perform the analysis without it. A comparison of the computational resources required and the results obtained with and without conformal mapping could be a productive area for future research.

ACKNOWLEDGMENTs. The authors would like to express their appreciation to Mr. Rodolfo Bonacci, now with the Federal Communication Commission, for his extensive help with this project.

\section{REFERENCES}

Buck, N. L., and G. P. Groff. 1995. Determining the thickness of layers of materials by dielectric constant measurements. Transactions of the ASAE 38(6): 1903-1910.

Chang, C. N., and J. F. Cheng. 1993. Hybrid quasistatic analysis of multilayer microstrip lines. IEE Proceedings-H 140(2): 79-83.

Churchill, R. V. 1960. 2nd Ed. Complex Variables and Applications. New York, N.Y.: McGraw-Hill.

Dongarra, J. J., J. R. Bunch, C. B. Moler, and G. W. Stewart. 1979. LINPACK Users' Guide. Philadelphia, Pa.: Society for Industrial and Applied Mathematics.

Hayt, W. H. 1989. Engineering Electromagnetics. New York, N.Y.: McGraw-Hill.

Institute of Electrical and Electronic Engineers. 1972. IEEE Standard Dictionary of Electrical and Electronics Terms. New York, N.Y.: John Wiley \& Sons.

Kapoor, S., and J. B. Schneider. 1995. Characterization of microstrip discontinuities using conformal mapping and the finite-difference time-domain method. IEEE Transactions on Microwave Theory and Techniques 43(11): 2636-2639.

Ramo, S., J. R. Whinnery, and T. Van Duzer. 1967. Fields and Waves in Communication Electronics. New York, N.Y.: John Wiley \& Sons.

Sylvester, P. P., and R. L. Ferrari. 1990. 2nd Ed. Finite Elements for Electrical Engineers. New York, N.Y.: Cambridge University Press. 